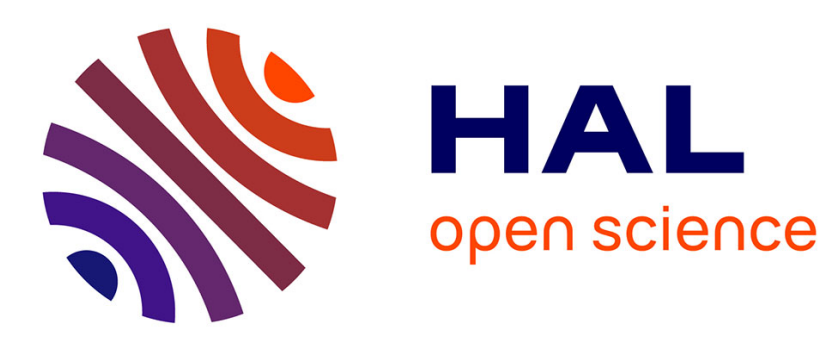

\title{
Vers une modélisation de la dynamique intra urbaine
} Denise Pumain, Thérèse Saint-Julien, Lena Sanders

\section{To cite this version:}

Denise Pumain, Thérèse Saint-Julien, Lena Sanders. Vers une modélisation de la dynamique intra urbaine. Espace Géographique, 1984, 13 (2), pp.125-135. 10.3406/spgeo.1984.3912 . halshs-01488353

\section{HAL Id: halshs-01488353 \\ https://shs.hal.science/halshs-01488353}

Submitted on 13 Mar 2017

HAL is a multi-disciplinary open access archive for the deposit and dissemination of scientific research documents, whether they are published or not. The documents may come from teaching and research institutions in France or abroad, or from public or private research centers.
L'archive ouverte pluridisciplinaire HAL, est destinée au dépôt et à la diffusion de documents scientifiques de niveau recherche, publiés ou non, émanant des établissements d'enseignement et de recherche français ou étrangers, des laboratoires publics ou privés. 


\title{
Vers une modélisation de la dynamique intra urbaine Mme Denise Pumain, Thérèse Saint-Julien, Lena Sanders
}

\section{Résumé}

La modélisation spatiale et dynamique des structures intra-urbaines connaît des renouvellements importants liés à l'utilisation des formulations mathématiques tirées de la théorie des catastrophes ou de la théorie des structures dissipatives. Ces modèles reprennent les bases traditionnelles de la théorie urbaine en y introduisant une dynamique qui considère la structure spatiale et son évolution non plus comme un donné mais comme le résultat d'interactions complexes. L'exploration des rapports entre la forme mathématique de ces interactions et la forme qualitative des configurations urbaines ainsi construites est en cours, aussi bien par des expérimentations théoriques que par des simulations d'histoires urbaines observées.

\begin{abstract}
Toward a modelling of intra-urban dynamics. - Modelling of intra-urban spatial dynamics has recently witnessed some important innovative impulses from catastrophe theory as well as from theories on dissipative structures. These models resonate with traditional urban theory and add a new dynamic element which considers the evolution of spatial structures not just as taken-for-granted fact, but rather as the product of complex interactions. A series of theoretical experiments, coupled with simulations based on observations from urban history is now under way, aiming to unravel connections between the mathematical form of urban interactions and the qualitative form of urban configurations.
\end{abstract}

\section{Citer ce document / Cite this document :}

Pumain Denise, Saint-Julien Thérèse, Sanders Lena. Vers une modélisation de la dynamique intra urbaine. In: Espace géographique, tome $13, n^{\circ} 2,1984$. pp. 125-135;

doi : $10.3406 /$ spgeo.1984.3912

http://www.persee.fr/doc/spgeo_0046-2497_1984_num_13_2_3912

Document généré le 06/09/2016 


\title{
VERS UNE MODÉLISATION DE LA DYNAMIQUE INTRA-URBAINE
}

\author{
Denise PUMAIN, Thérèse SAINT-JULIEN, Léna SANDERS
}

Université de Paris-I

GEOGR. THEORIQLE GEOOGR. LRBAANF MODELISATTION

MOI)ELI,INC THEORETICAL GEOGR. LRBAN GEOGR
RÉSUMÉ. - La modélisation spatiale et dynamique des structures intra-urbaines connaît des renouvellements importants liés à l'utilisation des formulations mathématiques tirées de la théorie des catastrophes ou de la théorie des structures dissipatives. Ces modèles reprennent les bases traditionnelles de la théorie urbaine en y introduisant une dynamique qui considère la structure spatiale et son évolution non plus comme un donné mais comme le résultat d'interactions complexes. L'exploration des rapports entre la forme mathématique de ces interactions et la forme qualitative des configurations urbaines ainsi construites est en cours, aussi bien par des expérimentations théoriques que par des simulations d'histoires urbaines obser. vées.

ABSTRACT. - Toward a modelling of intra-urban dynamics. - Modelling of intra-urban spatial dynamics has recently witnessed some important innovative impulses from catastrophe theory as well as from theories on dissipative structures. These models resonate with traditional urban theory and add a new dynamic element which considers the evolution of spatial structures not just as taken-for-granted fact, but rather as the product of complex interactions. A series of theoretical experiments, coupled with simulations based on observations from urban history is now under way, aiming to unravel connections between the mathematical form of urban interactions and the qualitative form of urban configurations.

"Moi aussi jai pensé à un modèle de ville duquel je déduis tous les autres... C'est une ville qui n'est faite que d'exceptions, d'impossibilités, de contradictions. d'incongruités, de contre-sens. Si une ville ainsi faite est tout ce quilly a de plus improbable en abaissant le nombre des éléments anormaux la probabilité grandit que la ville existe véritablement. Par conséquent, il suffit que je soustraie de mon modèle des exceptions, et de quelque manière que je procède jarriverai devant l'une des villes qui, quoique toujours par exception, existent. Mais je ne peux pas pousser mon opération plus loin qu'une certaine limite : jobtiendrai des villes trop vraisemblables pour être vraies. "

Italo CAMTNo, Les Villes Incisibles. Paris, Le Seuil, 1974.

Il n'y a guère, la construction et l'expérimentation de modèles du développement urbain ne suscitaient que scepticisme, voire ennui : la phase de croissance urbaine qui appelait à la création d'outils pour la maitriser est passée, les grandes et coûteuses machines de simulation trop gourmandes en données ont été abandonnées, si bien qu'en 1982 encore P.H. Derycke pouvait intituler la conclusion d'un des chapitres de son livre: "La crise des modèles urbains ". Et pourtant! De 
grands noms de la géographie - ou de la physique - se sont mis à élaborer de nouveaux modèles, la magie des mots "théorie des catastophes" ou "structures dissipatives" a réveillé les intérêts, et un peu partout dans le monde des équipes se forment pour tester ces outils tout neufs. Une mode de plus? ou un progrès significatif dans la longue histoire de la modélisation urbaine? Sans prétendre résoudre vraiment la question, les propos qui suivent tentent de préciser en quoi résident la nouveauté et les promesses des applications les plus récentes de la dynamique des systèmes à l'étude de l'évolution des structures intra-urbaines.

Hasard ou nécessité, le moment nous semble particulièrement opportun pour un renouveau de ce type de recherches. La croissance urbaine s'essouffle dans tous les pays industrialisés et l'on a vite fait de parler de "déclin des villes" ou de " crise urbaine". Les signes parfois spectaculaires qui ont récemment retenu l'attention sont cependant moins l'indice de la "fin des villes" que de l'actuelle modification des interactions qui s'établissent entre les hommes, les activités et l'espace, et qui sont créatrices de l'urbain. Historiquement, on a pu démontrer à quel point, dans un contexte de coûts de transports élevés pour les marchandises, les hommes et l'information, la concentration urbaine avait été le modèle privilégié par l'ensemble des acteurs urbains. Ces conditions initiales ont changé, du fait de transformations intervenues dans la sphère des techniques et aussi dans celle des mentalités. Les contraintes de contiguïté se sont inégalement amenuisées. Dans certains domaines, comme celui des transports de l'information, ce changement est aujourd'hui considéré comme un début de révolution. Par ailleurs le déclin démographique entraîne son cortège de problèmes économiques et sociaux. Il devient alors urgent de comprendre comment se met en place, sous nos yeux, une nouvelle ville, expression très vivante d'un corps social en profonde mutation, et d'essayer d'entrevoir ce que seront ses expressions les plus probables. Pour ce faire, plusieurs problèmes doivent être surmontés: d'ordre conceptuel, car nous avons à nous interroger sur la validité des cadres théoriques à l'intérieur desquels se situe encore aujourd'hui l'essentiel de la réflexion urbaine; d'ordre méthodologique, car prévoir le devenir urbain pour le préparer, puis le gérer, passe par la maîtrise d'outils de modélisation capables de tenir compte de la dynamique du système urbain et de l'espace de cette dynamique. Il faut explorer le champ des effets totalement contre-intuitifs dont sont porteuses les transformations en cours, en particulier dans la dimension spatiale.

Une nouvelle génération de modèles se prête particulièrement bien à cette exploration des structures spatiales, nées de mécanismes d'interaction multiples entre les activités et les habi- tants d'une ville. Les deux principales familles de modèles ont été élaborées indépendamment, l'une en Grande-Bretagne à l'Université de Leeds par l'équipe que dirige Alan Wilson, et l'autre à l'Université Libre de Bruxelles, par une équipe du département de chimie-physique du professeur Prigogine sous la direction de Peter Allen.

La principale caractéristique qui différencie les modèles d'Allen et de Wilson de ceux de leurs prédécesseurs est qu'il s'agit de modèles à la fois dynamiques et spatiaux. Certains des modèles classiques, tel celui de Lowry (1964) et ses dérivés, sont des modèles spatiaux non dynamiques: l'objet du modèle est de calculer, sous des contraintes données, quelle répartition de la population parmi un ensemble de zones s'ajusterait à une distribution donnée d'emplois de base, compte tenu également des emplois supplémentaires induits par cette population. Batty (1976) a introduit dans ce type de modèles une quasi-dynamique en simulant artificiellement l'effet d'induction décroissant dans le temps d'une certaine quantité d'emplois de base introduits à un moment donné. Le modèle de dynamique urbaine de J. Forrester (1969) est défini pour une unité urbaine prise dans sa totalité, sans subdivision spatiale interne. Le choix des variables dans ce modèle est d'ailleurs fonction de critères fonctionnels et temporels, mais nullement spatiaux (ainsi les entreprises classées en trois groupes: nouvelles, mûres et en déclin, les logements et les emplois classés en trois niveaux de qualité ou de revenu). Les rares interactions spatiales définies (migrants...) le sont pour les relations entre la ville et son environnement, mais pas à l'intérieur de la ville. Certes, l'une des applications du modèle de Forrester réalisées en France a tenté de remédier à cette insuffisance (Laudet, Fournier, 1978, Fournier, 1983) en considérant deux zones, la ville-centre et la banlieue, à l'intérieur de l'agglomération de Carpentras, et en ajoutant au modèle des interactions entre les deux zones. Mais la manière dont est construit le modèle de Forrester rendrait très complexe et difficile la simulation de l'évolution pour un plus grand nombre de zones.

L'apport des modèles d'Allen et de Wilson ne se réduit pas à une simple extension des modèles préexistants combinant les dimensions spatiale et temporelle. Le type de formalisation mathématique qu'ils utilisent pour engendrer une dynamique leur permet de provoquer des modifications qualitatives de la structure spatiale des villes. Là réside la véritable innovation. Les notions d'équilibre dynamique, de changement de structure, de discontinuité liée à une évolution sont connues depuis longtemps. Mais la possibilité de traduire en langage mathématique et de produire par simulation, sans intervention extérieure ces modifications qualitatives, est toute récente. Lorsque des interactions entre des variables ont été mesu- 
rées, on est capable désormais d'indiquer pour quelle valeur de tel ou tel paramètre la configuration réalisée est susceptible de basculer vers une autre forme d'organisation. Il devient alors possible de mieux comprendre "la logique des villes", de déterminer pourquoi, parmi un très grand nombre de configurations possibles, certaines sont plus souvent que d'autres réalisées, de voir comment sont engendrées des situations irréversibles et d'explorer l'univers des futurs possibles à partir d'une histoire urbaine donnée, en attribuant à chacun une certaine probabilité.

Avant de préciser le contenu de la démarche de chacun de ces deux types de modèles, nous rappellerons sur quels principes fondamentaux de la théorie urbaine ils sont construits. Ces principes ne sont pas nouveaux; l'insuffisance de leurs pouvoirs descriptif et explicatif en regard des observations empiriques est bien connue. On peut espérer cependant que ces modèles contribueront à améliorer l'état de nos connaissances en ce domaine, puisqu'ils vont nous permettre d'expérimenter ces éléments de théorie dans un contexte spatial et temporel caractérisé de manière beaucoup plus réaliste qu'auparavant.

\section{LES BASES THÉORIQUES REPRISES PAR LES DEUX MODÊLES}

Quelque sophistiqués que soient ces modèles dans leur formulation mathématique et quelque nuancés qu'ils soient dans leurs applications, ils n'en reposent pas moins, comme leurs prédécesseurs, sur quelques fondements extrêmement simples. Ces fondements définissent la nature des interactions reliant les variables essentielles d'un système urbain dans le domaine économique, spatial et temporel; leur application concourt à concrétiser une certaine image de la ville.

\section{Les interactions entre activités : le mécanisme d'induction}

Le principe bien connu de la base économique découle de l'hypothèse qu'il existe une relation multiplicative, constante dans un contexte donné, entre, d'une part, le nombre des emplois $\mathrm{E}$ et la population ${ }^{P}$ 'une ville

$$
\mathrm{P}=\alpha \mathrm{E}
$$

$\alpha$ étant l'inverse du taux d'activité de la population; et, d'autre part, entre la population $\mathrm{P}$ et le nombre des emplois dans les activités de service qui lui sont destinés $\mathrm{S}$ :

$$
S=\beta P
$$

$\beta$ étant le taux d'induction d'emplois de service par la population. L'emploi total $\mathrm{E}$ est la somme de ces emplois de service $S$ et de l'emploi $E^{\text {b) }}$ dans les activités de base qui, répondant à une demande extérieure à la ville, lui assurent des ressources lui permettant de se procurer les biens et les services qu'elle ne produit pas elle-même.

$$
\mathbf{E}=\mathrm{E}^{\mathrm{b}}+\mathrm{S}
$$

Ces trois équations décrivent des relations linéaires simples entre population, emplois fondamentaux et emplois de service. En substituant les termes, on peut obtenir l'expression de la population et du nombre total d'emplois en fonction des seuls emplois fondamentaux :

$$
E=\frac{E^{b}}{1-\alpha \beta} \text { et } P=\frac{\alpha E^{b}}{1-\alpha \beta}
$$

L'expression $1 /(1-\alpha \beta)$ est le multiplicateur de la base économique, il intègre à la fois les effets directs et indirects du secteur fondamental sur les autres activités urbaines.

Le plus souvent, dans les modèles intra-urbains, les activités ne sont pas classées strictement d'après le critère pur de la base économique - activités exportatrices et activités induites mais d'après leurs contraintes de localisation: activités de services à la population et aux industries d'une part, dont la localisation est calculée de manière endogène, et les autres activités d'autre part, dont la localisation est souvent imposée de manière exogène.

Lowry fut le premier à utiliser un tel mécanisme en liaison avec un modèle d'interaction spatiale. Selon une procédure itérative, ce modèle calcule la distribution de la population totale et des emplois dans les différentes parties d'une région en l'ajustant à la distribution des emplois de base, donnée une fois pour toutes. Les valeurs alors obtenues pour les paramètres permettent éventuellement de faire des projections pour évaluer l'effet de l'implantation, à tel ou tel endroit, de nouveaux emplois de base.

Le mécanisme d'induction décrit par la théorie de la base économique s'applique bien à des sous-ensembles spatiaux relativement fermés (par exemple différentes villes dans un système régional). Son interprétation est plus difficile dans le cadre des différents quartiers d'une agglomération urbaine où l'exigence de proximité entre l'offre d'emplois et la résidence de la population occupant ces emplois est relativement plus faible qu'à l'échelon d'un système de villes.

Enfin nous ne reviendrons pas sur les difficultés pratiques associées à la mise en œuvre de cette théorie (voir entre autres Pumain, SaintJulien, 1976), du fait de l'inadéquation des nomenclatures d'activités aux critères de localisation requis pour l'appliquer, difficultés auxquelles 
n'échappent pas les tentatives d'application de ces modèles.

\section{Les interactions spatiales: l'effet dissuasif de la distance}

Pour calculer l'importance des flux de personnes entre deux quartiers urbains (afin d'en déduire ensuite une demande potentielle en emplois, en services, en logements ou en infrastructures de transports), on utilise très généralement des modèles d'interaction de type gravitaire. L'hypothèse est que le volume des déplacements entre deux zones est proportionnel au produit des masses de chaque zone et est une fonction décroissante de la distance qui les sépare. Parmi les nombreuses formulations qui ont été proposées (Wilson, 1970), celle qui apparait le plus souvent dans les modèles dynamiques est la suivante:

$$
\mathrm{T}_{i j}=\frac{\mathrm{P}_{\mathrm{i}} \mathrm{W}_{j}^{\alpha} \mathrm{e}^{-\beta c_{i j}}}{\sum_{j} \mathrm{~W}_{j}^{\alpha} \mathrm{e}^{-\beta c_{i j}}}
$$

avec $\mathrm{T}_{\mathrm{ij}}=$ nombre d'individus se déplaçant de $\mathrm{i}$ vers $\mathrm{j}$.

$\mathbf{P}_{\mathrm{i}} \quad=$ nombre d'individus de la zone $\mathrm{i}$ susceptibles de se déplacer.

$\mathrm{W}_{\mathrm{j}}=$ attractivité de la zone $\mathrm{j}$.

$\mathrm{C}_{\mathrm{ij}}=$ coût de transport de $\mathrm{i}$ à $\mathrm{j}$.

$\alpha, \beta=$ paramètres du modèle.

Ce modèle, ou des variantes répondant à diverses contraintes, est utilisé par exemple pour calculer le flux quotidien $\mathrm{T}_{\mathrm{ij}}$ entre une zone d'emploi $i$ et une zone de résidence $j$, ou pour décrire la fréquentation par la population des zones i d'un ensemble de services dont la distribution spatiale est connue (zones j). Le dénominateur traduit la concurrence exercée par l'ensemble des zones en matière d'attractivité. Le rapport

$$
\frac{\mathrm{W}_{j}^{a} \mathrm{e}^{-\beta c_{j}}}{\sum_{j} \mathrm{~W}_{j}^{a} \mathrm{e}^{-\beta c_{i 1}}}
$$

représente donc la probabilité pour un individu travaillant en $\mathrm{i}$ de résider en $\mathrm{j}$ (ou, résidant en $\mathrm{i}$, de rechercher un service en j). En multipliant cette probabilité par la population active travaillant (résidant) en $i$, on obtient le flux de déplacements liés au travail (ou à la fréquentation commerciale) $\mathrm{T}_{\mathrm{i} j}$.

Dans ce modèle, $W_{j}$ est un indicateur de taille ou de masse (auquel on peut combiner des indicateurs de qualité ou de niveau) de la zone attractive. Un paramètre $\alpha=1$ correspond à une attractivité proportionnelle à cette masse; si $\alpha<1$, l'attractivité croît moins vite que la masse (exprimant la sensibilité à la congestion ou aux déséconomies d'échelle); si $\alpha>1$, l'attractivité augmente plus vite que la taille (sensibilité aux économies d'agglomération, à la variété plus grande associée à l'effet de taille).

Le paramètre $\beta$ représente la sensibilité des déplacements aux coûts induits par la distance. Ces deux paramètres représentent donc chacun des caractéristiques essentielles du comportement spatial des populations.

\section{La dynamique des populations}

En l'absence de limites de ressources, une population $\chi$ varie en fonction du temps $\mathrm{t}$ selon une loi exponentielle : $\chi=K \cdot e^{\mathrm{ut}}, \mathrm{K}$ étant une constante, et le paramètre $u$ indiquant le sens de l'évolution: croissance s'il est positif, déclin s'il est négatif.

Les modèles urbains, rapportant la croissance des variables qu'ils considèrent à des espaces limités, utilisent tous des fonctions du temps qui traduisent l'intervention de contraintes (saturation, déséconomies d'échelle...), et ralentissent la progression exponentielle. Le modèle de Forrester distingue ainsi, à côté des variables de stock qui décrivent l'état du système à un moment donné, des variables de flux qui sont des taux de variation agissant d'un instant à l'autre sur le niveau des variables de stock. Ces taux de variation ne sont pas tenus constants mais varient en fonction du niveau atteint par une ou plusieurs variables de stock, en général d'après une "fonction tabulée " qui traduit des rétroactions d'une variable sur une autre.

Dans les modèles d'Allen et de Wilson, la forme même des équations s'inspire de celle de la loi logistique. Celle-ci décrit la variation par unité de temps $\mathrm{dx} / \mathrm{dt}$ d'une population $\mathrm{x}$ dont la survie dépend d'une capacité de ressources limitée à $\mathrm{N}$ :

$$
\frac{d x}{d t}=u \cdot\left(1-\frac{x}{N}\right) \cdot x
$$

où $u$ est la vitesse d'ajustement de la population entre son niveau à un moment donné et le niveau potentiel qu'elle peut atteindre, tel qu'il est limité par N.

Dans un système humain, la capacité $\mathrm{N}$ n'est cependant pas fixée une fois pour toutes. Il existe des effets de rétroaction entre la population et les facteurs limitant son développement, tels que le niveau de ceux-ci peut s'élever au fur et à mesure que la population s'accroît (fig. 1). Dans l'équation, $\mathrm{N}$ devient dépendant de $\mathrm{x}$ à travers un ou plusieurs paramètres $\lambda$ :

$$
\frac{\mathrm{dx}}{\mathrm{dt}}=\mathrm{u} \cdot\left(1-\frac{\mathrm{x}}{\mathrm{N}\left(\mathrm{x}, \lambda_{\mathrm{i}}\right)}\right) \cdot \mathrm{x}
$$

Les modèles urbains d'Allen et de Wilson ont adapté cette formulation à la dynamique des populations urbaines, en assimilant la capacité 
d'une zone à son potentiel d'emploi, et en rendant celui-ci dépendant non seulement du niveau déjà atteint dans la zone considérée, mais aussi de la situation relative de celle-ci par rapport à l'ensemble des autres zones de l'agglomération. La nouveauté de ces modèles réside donc dans la combinaison de trois mécanismes d'origine différente: un principe écologique de croissance, un principe géographique d'interaction spatiale, un principe économique d'induction rassemblés dans un contexte de compétition pour l'espace et d'organisation spatiale d'une agglomération.

\section{Une certaine image de la ville}

Quelle interprétation de la ville résulte de l'insertion de ces principes théoriques dans la dynamique des systèmes ouverts situés loin de l'équilibre? Dans chacun des modèles, la ville est considérée comme un territoire ouvert sur l'extérieur, mais à l'intérieur duquel les interactions étudiées sont supposées être plus importantes qu'elles ne le sont avec les espaces situés à l'extérieur. Le moteur de l'évolution est donné par l'écart entre l'existant et le potentiel, lui-même en constante révision sous l'effet des variations internes et externes. Les acteurs ne tendent plus à optimiser une certaine fonction, à mettre le système en équilibre, mais l'ajustent en permanence à des conditions changeantes, ce processus intervenant lui-même dans l'évolution des conditions. Selon P. Allen (1981), les non-linéarités qui structurent une ville résulteraient avant tout du comportement d'imitation et d'anticipation des acteurs.

Cette interprétation est située dans le cadre d'une économie libérale, où le jeu de l'offre et de la demande et une relativement libre compétition pour l'espace demeurent des ressorts prépondérants de la dynamique urbaine. Les mécanismes d'interaction introduits dans le modèle jouent librement en tout point de l'espace et s'appliquent à l'ensemble de l'agglomération considérée. Cela ne signifie pas cependant que l'espace soit homogène et isotrope: les accessibilités des zones, facteurs importants dans le calcul des attractivités, y sont calculées d'après des distances qui peuvent être mesurées sur un réseau; des contraintes spécifiques à certains espaces peuvent être introduites dans chacun des modèles.

D'autres interprétations plus ou moins implicites sont indissociables de toute expérimentation de modèles spatio-temporels. Ainsi les auteurs n'échappent pas à l'ambiguïté inhérente aux nécessités de lagrégation spatiale: les paramètres et la forme des équations font référence au comportement des acteurs, et s'appliquent en fait à des agrégats de personnes ou d'activités regroupés dans une même subdivision de lagglomération.
Les mécanismes décrits jouent de manière continue sur un espace interne qui est cependant discrétisé, subdivisé en zones. Celles-ci n'ont pas à être nécessairement homogènes, mais leur diversité interne n'est pas prise en compte et les effets de transition intrazones sont ignorés. De même, le temps est découpé en intervalles pour le calcul, et des variations en principe simultanées sont calculées successivement.

Ces contraintes théoriques ne sont cependant pas d'une extrême gravité, en regard des contraintes liées à l'application pratique de ces modèles! Tous les praticiens de l'urbain sont suffisamment informés des limites des sources, en matière de variables et de découpage pertinents ou de séries temporelles, pour que nous n'insistions pas davantage sur ce point.

\section{LE MODĖLE D'A. WILSON}

A. Wilson a élaboré un modèle régional dynamique à partir des modèles classiques d'interaction spatiale. Des modèles simples de distribution des activités de services et de la population résidente sont dynamisés puis reliés l'un à l'autre, à travers des mécanismes du type Lowry, pour constituer un modèle général. Le point essentiel de cette approche est la «dynamisation » d'un modèle d'interaction simple. Nous reprenons ici la démarche de Wilson à propos du modèle de localisation des activités de services.

Le modèle classique de Wilson permet de calculer les flux $F_{\mathfrak{j}}$ (flux monétaires ou nombre de consommateurs) entre une zone d'origine $i$, lieu de résidence et une zone de destination $\mathrm{j}$, lieu où se trouve le service recherché :

$$
F_{i j}=\frac{P_{i} W_{j}^{a} e^{-\beta c_{11}}}{\sum W_{j}^{a} e^{-\beta c_{i n}}}
$$

où $W_{1}$ représente l'attractivité commerciale de la zone $\mathrm{j}$ et $\mathrm{P}$, le nombre de consommateurs résidant dans la zone i. Les autres termes de l'équation sont définis de la même manière que dans l'équation [4] ci-dessus. Connaissant la distribution de l'ensemble des flux à travers l'agglomération, il est facile d'obtenir le montant des achats effectués en j (ou la clientèle totale de j). Ce nombre représente la demande $D_{1}$ qui est induite par l'ensemble de l'agglomération dans la zone $\mathrm{j}$ et se calcule de la façon suivante:

$$
\mathrm{I}_{1}=\sum_{1} \mathrm{~F}_{11}
$$

Dans les modèles classiques, l'attractivité $W$, d'une zone $\mathrm{j}$ est une variable exogène, mesurée par la surface commerciale, le chiffre d'affaires ou le nombre de services offerts en $j$. Ce facteur caractérise donc l'offre en services et le modèle 
détermine la distribution des flux de consommateurs en fonction de cette offre, dont la distribution est donnée a priori. Le grand apport du dernier modèle de Wilson est de considérer l'offre comme une variable endogène et de modéliser son évolution dans le temps. Il suppose que les décideurs, c'est-à-dire les producteurs de services, ne sont pas indifférents à la répartition de la demande à travers l'agglomération et essaient de s'y adapter. Le système tend ainsi à évoluer vers un équilibre entre les coûts de production, fonction de l'attractivité $W_{j}$ et les revenus produits, fonction de la demande $D_{j}$.

Pour traduire ces hypothèses, Wilson choisit l'équation différentielle qui décrit la variation de $\mathrm{W}_{\mathrm{j}}$ par unité de temps dt:

$$
\frac{\mathrm{d} \mathrm{W}}{\mathrm{dt}}=\varepsilon\left(\mathrm{D}_{\mathrm{j}}-\mathrm{kW}_{\mathrm{j}}\right)
$$

où $\mathrm{k}$ est un paramètre convertissant les coûts de production associés à $W_{\mathrm{j}}$ dans la même unité que les revenus produits par $D_{j} ; \varepsilon$ traduit la vitesse avec laquelle l'offre s'ajuste à la demande. ple :

Cette équation décrit une dynamique très sim- si la demande est supérieure à l'offre $\left(D_{j}>\mathrm{kW}_{\mathrm{j}}\right)$ en une zone $\mathrm{j}$ de la région, le terme $\mathrm{D}_{\mathrm{j}}-\mathrm{kW}_{\mathrm{j}}$ est positif et la variation $\mathrm{dW} / \mathrm{dt}$ l'est donc également; l'offre $W_{j}$ tend alors à croître vers la demande $D_{j}$, potentiel induit dans la zone j par la demande de l'ensemble de la région;

- si la demande est au contraire inférieure à l'offre $\left(D_{i j}<k W_{j}\right)$, le terme $D_{j}-k W_{j}$ est négatif et la variation $\mathrm{dW}_{\mathrm{j}} / \mathrm{dt}$ également; l'offre tend alors à diminuer pour s'ajuster à une demande plus faible; plus la différence entre offre et demande est importante, plus le terme $D_{j}-k W_{j}$ est élevé en valeur absolue et plus la diminution de l'offre est accentuée;

- si l'offre correspond à la demande $\left(\mathrm{D}_{\mathrm{i}}=\mathrm{kW}_{\mathrm{j}}\right)$, le système est en équilibre et la variation $\mathrm{dW}_{\mathrm{j}} / \mathrm{dt}$ est nulle.

La forme de l'équation [9] traduit donc des hypothèses simples, mais le développement des termes qui la composent donne une idée de sa complexité et des difficultés qu'elle pose à une étude analytique. En effet, [7] et $[8] \Rightarrow$ :

$$
\mathrm{D}_{j}=\sum_{i} \mathrm{~F}_{\mathrm{ij}}=\sum_{i} \frac{\mathrm{P}_{\mathrm{t}} \mathrm{W}_{j}^{\alpha} \mathrm{e}^{-\beta c_{i}}}{\sum_{j} \mathrm{~W}_{j}^{\alpha} \mathrm{e}^{-\beta c_{i t}}}
$$

et (9) équivaut donc à :

$$
\frac{\mathrm{d} \mathrm{W}_{\mathrm{j}}}{\mathrm{dt}}=\varepsilon\left(\sum_{\mathrm{i}} \frac{\mathrm{P}_{1} \mathrm{~W}_{\mathrm{j}}^{\alpha} \mathrm{e}^{-\beta c_{1}}}{\sum \mathrm{W}_{j}^{\alpha} \mathrm{e}^{-\beta c_{i}}}-\mathrm{kW}_{\mathrm{i}}\right)
$$

Wilson a réussi à mettre en évidence les caractéristiques essentielles de la relation entre les variables $D_{j}$ et $W_{i}$; la figure 2 représente la forme générale de la fonction

$$
D_{j}=\frac{\sum_{i} P_{i} W_{j}^{\alpha} e^{-i c_{i j}}}{\sum_{j} W_{j}^{\alpha} e^{-\beta c_{i j}}}
$$

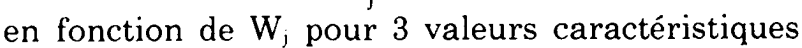
du paramètre $\alpha$.

L'état d'équilibre du système correspond à $\mathrm{D}_{\mathrm{j}}=\mathrm{kW}_{\mathrm{j}}$ et se situe donc à l'intersection entre ces courbes et la droite $\mathrm{D}_{j}=\mathrm{kW}$. La figure 3 en donne une représentation graphique pour deux exemples. Cet exemple montre le rôle joué par le paramètre $\alpha$, traduisant la sensibilité des consommateurs à l'importance en taille des centres de service.

$S i \alpha<1$, pour des valeurs faibles de l'attractivité $W_{j}$, une légère différence a des répercussions importantes au niveau de la demande; les producteurs ont donc intérêt à augmenter leur attractivité pour répondre à cette demande importante; l'équilibre est réalisé pour une valeur $W_{j}^{A}$ de l'at tractivité; au-delà de ce seuil, l'effet négatif produit sur les consommateurs par une taille trop importante l'emporte (cf. § I.2), et la demande reste inférieure à l'offre; pour maximiser son profit, le producteur a donc intérêt à rester proche de ce point d'équilibre.

Si $\alpha>1$, pour des valeurs faibles de l'attractivité, la demande est bien inférieure à l'offre; les producteurs n'ont donc aucun intérêt à s'agrandir et l'attractivité risque de rester proche de 0 ; au-delà du $1^{\cdots}$ point d'équilibre $W_{j}^{\wedge}$, au contraire, la courbe se situe au-dessus de la droite et la demande est donc supérieure à l'offre; l'intérêt des producteurs les entraîne donc à augmenter leur attractivité; au-delà du $2^{\prime \prime}$ point d'équilibre $\mathrm{W}_{\mathrm{j}}^{\mathrm{k}}$, l'offre est à nouveau supérieure à la demande et l'attractivité tendra à diminuer pour se rapprocher de la demande; on constate aisément que les situations des 2 points d'équilibre $\mathrm{A}$ et $\mathrm{B}$ ne sont pas symétriques: on tend toujours à s'écarter du premier (pour les valeurs inférieures à $W_{j}^{A}$, la demande dépasse l'offre, provoquant une diminution irréversible de l'attractivité et le mouvement contraire est observé pour les valeurs supérieures à $W_{i}^{\prime}$ ) et à se rapprocher du second; le point $\mathrm{A}$ est dit en équilibre instable alors que $\mathrm{B}$ caractérise un équilibre stable.

Ainsi, pour une valeur initiale donnée de l'attractivité $W_{\mathrm{i}}$ d'une zone $\mathrm{j}$, des évolutions très différentes peuvent apparaître selon les valeurs du paramètre $\alpha$. Les structures spatiales qui en résultent sont très différenciées : pour $\alpha<1$, une structure spatiale composée de nombreux centres de services de petites dimensions tend à s'organiser; pour $\alpha>1$, au contraire, une structure spatiale composée d'un nombre restreint de gros centres de services s'impose.

La valeur $\alpha=1$ constitue donc un seuil critique dans ce modèle et entraîne une bifurcation. Un léger ćcart de part et d'autre de cette valeur entraîne des structures spatiales qualitativement 
différentes alors que des écarts beaucoup plus importants autour de valeurs non critiques n'entraînent que des changements continus de type quantitatif. La figure 4 en donne un exemple: 3 courbes sont représentées, correspondant à 3 valeurs distinctes de $\alpha$, supérieures à 1 . Qualitativement, les courbes sont de même nature et traduisent les mêmes phénomènes. Seules diffèrent les valeurs des points d'équilibre, c'est-à-dire les seuils à partir duquel un centre en particulier tend à se développer ou à décliner. Plus $\alpha$ est grand, plus les consommateurs sont sensibles aux économies d'échelle associées aux centres de grande taille, et plus le développement des petits centres est entravé au profit des grands. Le même type de changement continu se produit pour des valeurs inférieures à 1 , et c'est seulement autour de cette valeur critique que l'on constate un véritable renversement de tendance.

L'équation différentielle [11] décrivant la dynamique du système contient en tout 4 paramètres : $\alpha, \beta, \varepsilon$ et $\mathrm{k}$. Un exemple de bifurcation vient d'être mis en évidence pour le paramètre $\alpha$, les autres paramètres du système restant constants. Mais la complexité de l'équation [11], avec ses nombreuses non-linéarités, offre de multiples possibilités de bifurcations. Aussi existe-t-il certaines configurations critiques où le moindre changement dans un paramètre peut changer fondamentalement la dynamique du système, alors que des changements dans d'autres configurations se traduisent simplement par des variations continues.

Wilson utilise un deuxième modèle d'interaction simple pour déterminer les localisations de la population résidente à partir des lieux de travail. Il procède par analogie avec le modèle développé ci-dessus et traduit par une équation différentielle l'évolution de l'offre de logement vers un équilibre avec la demande. Les deux sous-modèles sont liés par les mécanismes de Lowry: la localisation des emplois de base est donnée de façon exogène. Le modèle de localisation de la population résidente détermine en premier lieu la répartition de la population résidente associée à ces emplois de base. Cette population a des besoins en services et induit donc des emplois dans ce secteur dont la distribution est donnée par le modèle des activités de services développé ci-dessus. Les nouveaux emplois induisent une population résidente sup. plémentaire qu'il faut localiser et le premier modèle est de nouveau utilisé. Ainsi, par itérations successives et par passage d'un sous-modèle à l'autre, les emplois et la population résidente sont répartis entre les différentes zones de la région étudiée.

A notre connaissance, ce modèle complet n'a pas encore été testé par Wilson. En revanche. chacun des deux sous-modèles a été appliqué à des cas théoriques et de nombreuses simulations ont été effectuées pour étudier les rôles précis
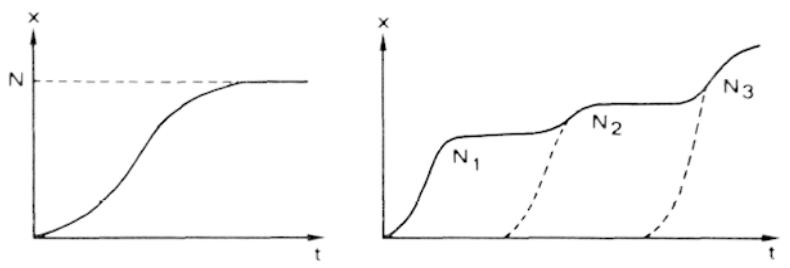

Fici. 1. - Croissance logistique d'une population selon sa capacité limite $N$.

(D’après Prigogine, Stengers, 1979, p. 183-184).
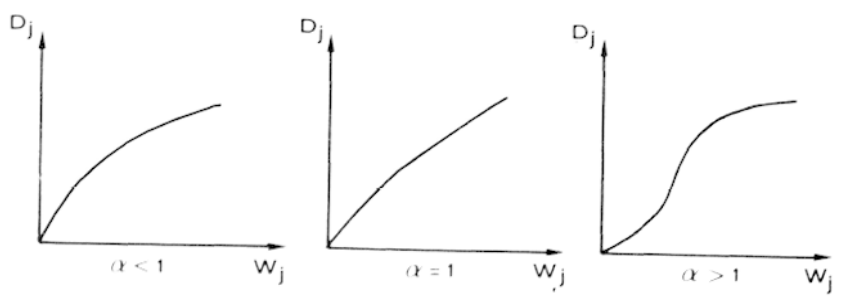

Fıı:. 2. - Effet de la sensibilité des consommateurs à la taille des centres $(\alpha)$ sur la relation entre l'offre $\left(W_{j}\right)$ et la demande $\left(D_{f}\right)$ de services.
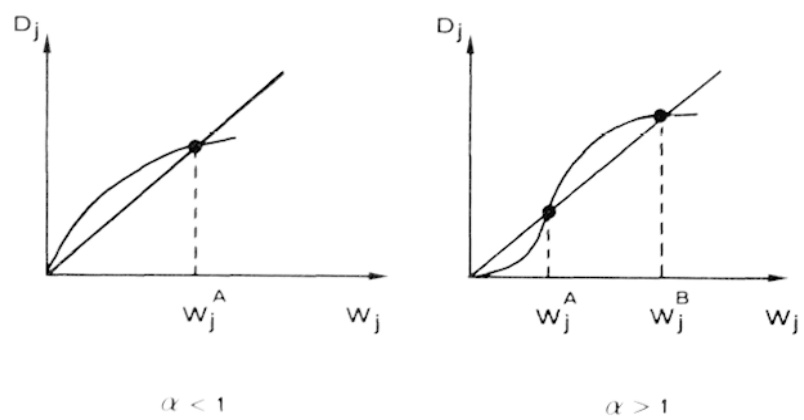

FIc. 3. - Points d'équilibre stable (B) et instable (A) entre l'offre $\left(W_{i}\right)$ et la demande $\left(D_{j}\right)$ de services.

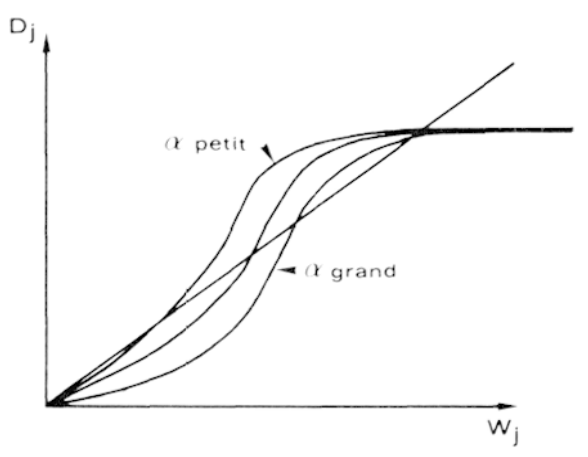

Fısi. 4. - Effet de la sensibilité des consommateurs à la taille des centres ( $\alpha$ ) sur les seuils de taille intervenant dans le développement de ces centres. 
joués par les différents paramètres. Pour mieux rendre compte de la complexité de la réalité et des différences de comportements spatiaux, les variables du modèle sont désagrégées par groupe de revenus, type de logements, type d'activités, etc. Les simulations donnent des résultats très intéressants (Clarke et Wilson, 1983a et b), mais est-il possible de conclure sur la validité de ces modèles avant qu'une expérimentation pratique ait été menée à bien?

\section{LE MODĖLE DE P. ALLEN}

Alors que Wilson s'est référé à des modèles urbains classiques dans lesquels il a introduit une dynamique, P. Allen a construit directement un modèle urbain dynamique à partir des équations utilisées en physique-chimie puis en biologie pour décrire l'apparition de phénomènes d'auto-organisation dans des systèmes ouverts situés loin de l'équilibre.

Ce modèle considère ainsi l'espace d'une ville comme un système ouvert, subdivisé en zones. Chaque zone est caractérisée par une certaine quantité d'emplois dans quatre types d'activités (activités industrielles et tertiaires dépendant d'une demande extérieure, activités de services à la population de courte et moyenne portée), par une certaine quantité de population résidente (ouvriers et cols blancs) et par une localisation et une accessibilité aux réseaux de transport et d'information.

Ce modèle se compose d'équations différentielles non linéaires qui décrivent chacune la variation par unité de temps de l'une des variables (quantité d'emplois ou de population d'un certain type) pour chaque zone de l'agglomération. Ces équations utilisent la formulation de la croissance logistique, pour la population comme pour l'emploi dans une zone.

La variation du nombre d'emplois $S_{i}^{k}$ de type $k$ dans une zone donnée i dépend de l'écart entre le niveau d'emploi déjà atteint et le niveau potentiel $\mathrm{E}_{\mathrm{i}}^{\mathrm{k}}$ qu'elle est susceptible d'atteindre :

$$
\frac{\mathrm{d} S_{i}^{k}}{\mathrm{dt}}=\alpha\left[1-\frac{\mathrm{S}_{i}^{\mathrm{k}}}{\mathrm{E}_{\mathrm{i}}^{\mathrm{k}}}\right] \mathrm{S}_{i}^{\mathrm{k}}
$$

où $\alpha$ représente la vitesse d'ajustement de l'offre d'emplois en i à l'emploi potentiel dans cette zone. Ce potentiel dépend de la demande extérieure pour les activités exportatrices ou d'une demande induite par l'agglomération pour les activités de services à la population. Le premier type d'activité est déterminé en quantité de manière exogène (par la demande extérieure $D^{k}$ ) et sa répartition dans l'agglomération dépend de l'attractivité que chaque zone peut exercer sur ce type d'activité. Le second type d'activité est déterminé, dans son volume et dans sa localisation, par les caractéristiques de chaque zone. Dans ce cas, la demande $D_{j}^{k}$ induite par chaque zone $\mathrm{j}$ de l'agglomération est fonction de sa population $\mathrm{x}_{j}$ et de la demande moyenne $\beta^{k}$ d'un individu pour le produit $k$ et s'écrit $D_{i}^{k}=\beta^{k} x_{j}$. Mais, selon toute vraisemblance, la zone i n'est pas la seule à offrir le bien ou le service de type $\mathrm{k}$. Elle ne retiendra donc, de la demande totale exprimée pour ce service par la population d'une zone, qu'une fraction correspondant à son attractivité relative par rapport à l'ensemble de toute les autres zones de l'agglomération susceptibles d'offrir ce service.

Le potentiel d'emploi $E_{i}^{k}$ de la zone i s'écrit donc, pour les activités exportatrices :

$$
E_{i}^{k}=D^{h} \frac{A_{i}^{k}}{\sum_{j^{\prime}} A_{j}^{k}}
$$

où $\mathrm{A}_{\mathrm{i}}$ désigne l'attractivité de la zone i pour l'activité de type $\mathrm{k}$ et où le dénominateur $\sum \mathrm{A}_{j}^{\mathrm{k}}$ caractérise la concurrence exercée par l'ensemble de l'agglomération; pour les activités induites :

$$
\mathrm{E}_{\mathrm{i}}^{\mathrm{k}}=\sum_{j^{\prime}} \beta^{\mathrm{k}} \mathbf{x}_{\mathrm{j}} \frac{\mathrm{A}_{\mathrm{j}}}{\sum_{\mathrm{j}} \mathrm{A}_{\mathrm{j}^{\prime} \mathrm{j}}}
$$

où $\mathrm{A}_{\mathrm{ij}}$ représente l'attractivité exercée par i sur la clientèle de $j$; le terme $\sum_{j} A_{j j}$ exprime la concurrence de l'ensemble des 'zones $\mathrm{j}^{\prime}$ de l'agglomération sur la clientèle de $\mathrm{j}$; en additionnant les emplois induits par chaque zone $j$, on obtient donc le potentiel $E_{i}^{k}$ de la zone $i$.

Plusieurs éléments interviennent dans la mesure de l'attractivité $A_{i j}$ d'une zone i sur une zone j. La forme générale étant à peu près semblable pour les deux types d'activité, nous n'expliciterons ici que celle utilisée pour les services induits par la population: a) l'importance que cette attractivité a déjà en i joue positivement sur l'attractivité selon un paramètre $\rho^{k}$ qui mesure la propension de ce type d'activité à s'agglomérer et négativement selon un paramètre $\psi^{k}$ qui introduit l'effet des déséconomies d'agglomération; $b$ ) la distance $d_{i j}$ entre $i$ et $j$ intervient de façon négative dans l'attractivité; la sensibilité des individus à cette distance est mesurée par un paramètre $\Phi$, d'autant plus élevé que la distance joue un rôle dissuasif sur les déplacements des individus; $c$ ) l'espace disponible dans la zone i intervient également dans son attractivité; le terme est proportionnel à la densité maximale admise $\tau^{\mathrm{k}}$ pour l'activité de type $\mathrm{k}$ et inversement proportionnel à la densité de population résidente et d'activités existant déjà.

Ces trois termes interviennent simultanément dans la mesure de l'attractivité $A_{i}$ dont l'expression mathématique est la suivante :

$$
A_{i j}^{k}=\frac{1+\rho^{k} S_{j}^{k}\left(1-\psi^{k} S_{j}^{k}\right)}{1+\Phi^{k} d_{i j}} \cdot \frac{\tau^{k}}{\tau^{k}+\sum_{k} x_{j}^{k}+\sum_{1} S_{j}^{1}}
$$




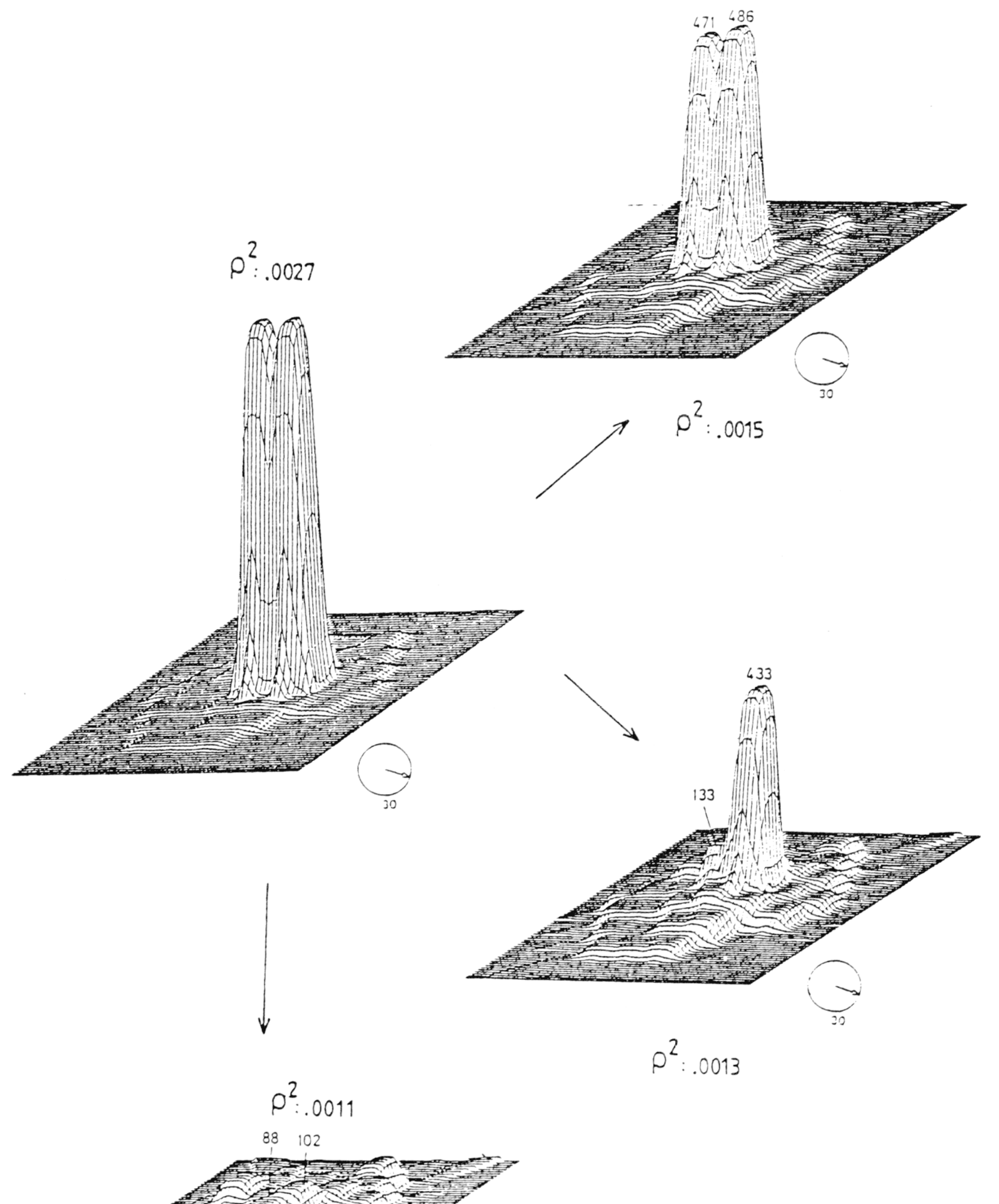

Fıı. 5. - Diversité des configurations spatiales obtenues selon les valeurs d'un paramètre (d'après Engelen et al., 1983).

Le paramètre $\mathrm{p}$ représente la propension d'une activité à s’agglomérer. 
Chaque attractivité ainsi définie est affectée d'un exposant qui traduit le degré d'homogénéité des préférences territoriales parmi les acteurs. Des équations analogues sont utilisées pour décrire l'évolution de chaque catégorie de population résidente (ouvriers et cols blancs). I.a variation dans une zone $\mathrm{j}$ est fonction de la quantité de résidents de chaque catégorie, employés par les différents types d'activité dans chaque zone $\mathrm{j}^{\prime}$, pondérée par l'attrait relatif qu'exerce la zone $j$ comme lieu de résidence pour les personnes travaillant dans la zone $j^{\prime}$. L'attrait relatif est le rapport entre l'attractivité que $\mathrm{j}$ exerce sur les actifs employés en j', rapporté à la somme des attractivités de toutes les zones.

L'attractivité résidentielle d'une zone j pour les employés d'une catégorie $\mathrm{k}$ (ouvriers ou cols blancs) travaillant dans une zone $\mathrm{j}^{\prime}$ est définie de façon similaire à l'attractivité économique exposée ci-dessus. Elle est fonction de la masse des résidents de cette catégorie en $\mathrm{j}$, de l'espace disponible dans la zone $\mathrm{j}$, et de la distance séparant $\mathrm{j}$ de $\mathrm{j}^{\prime}$ à travers un certain nombre de paramètres.

La formalisation mathématique de ces équations est relativement complexe, mais la décomposition des divers termes qui les forment montre, en fait, une série d'hypothèses qu'il est aisé d'expliciter d'une manière cohérente. Des modèles de dynamique territoriale de ce type ont été d'abord expérimentés dans des conditions théoriques, pour simuler la mise en place d'une hiérarchie de centres urbains (Allen, Sanglier, 1978) ou pour étudier le développement d'une ville fictive constituée d'une trame hexagonale de 19 zones (Allen et al. 1983) (fig. 5).

Nous avons, en collaboration avec l'équipe de P. Allen, expérimenté ce modèle pour simuler l'expansion récente de quelques agglomérations françaises (1). Le modèle suppose que des lois générales s'appliquent à une combinaison spatiale particulière, pour déterminer l'essentiel des transformations observées dans les distributions intra-urbaines de la population et des activités.

Dans un premier temps, le modèle a été calibré pour reproduire l'évolution observée de l'agglomération de Rouen (Pumain, Saint-Julien, Sanders, 1984) depuis 1954. Ce calibrage a favorisé différents approfondissements théoriques (forme des équations, nature, signification et mesure des paramètres). Il permet d'interpréter les écarts entre l'évolution simulée et réelle en termes de résidus par rapport à une dynamique d'ensemble. A plus long terme, la comparaison des paramètres

(1) Des applications du modèle de P. Allen sont en cours dans le cadre d'un contrat PIREN' du CNRS sur les agglomérations de Rouen, Nantes. Bordeaux et Strasbourg (par l'équipe P.A.R.I.S.) et à l'Université Libre de Bruxelles sur les régions hollandaises et l'agglomération de Bruxelles (par P. Allen, G. Engelen et M. Sanglier). calibrés sur l'évolution observée de plusieurs agglomérations, occupant un niveau comparable dans le réseau urbain français, doit permettre de mieux mesurer le degré de généralité des mécanismes d'évolution introduits dans le modèle, et donc de tester la pertinence de celui-ci en tant que modèle urbain opérationnel. Ce modèle pourrait en effet devenir un outil très utile pour éclaircir une série de questions très débattues aujourd'hui. Ainsi celle du déclin démographique des centres, celle du rôle de l'industrie dans la croissance et la structuration de l'espace intraurbain, celle des ségrégations résidentielles, ou encore celle des distances entre lieux d'habitat et de travail. A ces questions, le modèle n'apporte pas de réponse normative. Il devrait permettre cependant, sous différentes hypothèses, de prolonger les évolutions observées et de mesurer les conséquences, pas toujours intuitives, de certains choix des planificateurs sur la configuration ultérieure des villes.

\section{CONCLUSION}

De grandes similitudes apparaissent entre les modèles dynamiques de Wilson et d'Allen, tant sur le plan de la formalisation mathématique que des hypothèses économiques sous-jacentes. Il est difficile de comparer la valeur de ces modèles, car quelques caractéristiques fondamentales les différencient en rendant l'un ou l'autre plus attractif selon le cas. La forme des équations de Wilson permet une étude analytique des mécanismes de bifurcation. Il peut ainsi mettre en évidence de façon explicite des configurations critiques de paramètres et étudier le comportement du système autour de ses points d'équilibre. Cette démarche est extrêmement satisfaisante sur le plan théorique. Toutefois, un véritable test des potentialités de ce modèle ne peut résulter que de son application à une réalité observée. Des travaux sont faits dans ce sens par une équipe italienne (Lombardo, Rabino, 1983) et laissent espérer des résultats intéressants. On pourrait considérer le modèle d'Allen comme moins satisfaisant sur le plan de la théorie mathématique, la complexité de la formulation de l'attractivité rendant impossible toute approche analytique. En revanche, les possibilités d'applications concrètes le rendent très attractif pour les utilisateurs et les praticiens de l'espace urbain.

Une concertation plus large commence à s'établir entre les inventeurs des deux types de modèles et leurs utilisateurs (2). Une telle collabora-

(2) Un groupe de travail de I'IIASA, sous la direction de B. Johansson, a été constitué pour étudier la dynamique des aires métropolitaines. 
tion s'impose devant les difficultés liées à l'expérimentation, et l'état limité de nos connaissances empiriques comparatives des paramètres essentiels de la dynamique urbaine.

Il ne faut pas craindre de consacrer du temps et des moyens à ce qui peut n'apparaître dans un premier temps que comme un jeu un peu futile, que l'on $\mathrm{y}$ voie une application d'un formalisme de la dynamique, dont l'intérêt essentiel, d'ordre pédagogique, serait de "faire voir" les conséquences globales, prévisibles, d'un ensemble complexe d'interactions sur une structure spatiale, ou que l'on y voie un outil d'expérimentation de propositions théoriques pas très bien assises, en tout cas souvent contestées. La vraie question est qu'il n'existe pas, en sciences humaines, d'autre moyen que ce genre de simulation pour mettre à l'épreuve un corps de propositions tant soit peu global et complexe et susceptible de rendre compte des évolutions observées.

Manuscrit reçu en janvier 1984, révisé en février 198.1

\section{RÉFÉRENCES}

AI.EXANi)RE (C.), 1976, Contribution à une modélisation de la croissance $d u$ système urbain français 1945-1975. Université de Toulouse - Le Mirail, thèse de 3 " cycle, $272 \mathrm{p}$.

At.1.: (P.), 1981, "Self organization in complex systems ". Communication au colloque Thermodynamique et Sciences de l'Homme. Université de Créteil, 22-23 juin, $12 \mathrm{p}$.

Alt.k (P.), SAN(illtik (M.), 1979, «A dynamic model of growth in a central place system." Geographical Analysis, 11, p. 256-272.

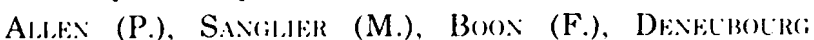
(J.L.), de PAlMin (A.), 1981. Models of urban settlement and structure as dinamic self-organizing systems. Washington. I).C.. Department of Transportation.

BNTY (M.) 1976. Urban modelling. Cambridge Lniversity Press, Cambridge.

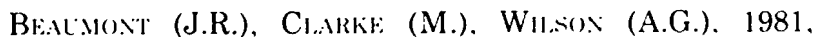
"The dynamics of urban spatial structure: some exploratory results using difference equations and bifurcation theory ". Environment and Planning $A$, 13.

Clarke (M.), WIL.soN (A.G.), 1983a, "The dynamics of urban spatial structure: progress and problems". Journal of Regional Science, vol. 13, no 1.

Clarke (M.), WILsoN (A.G.), 1983b. "Exploring the dynamics of urban housing structure : a 56 parameter residential location and housing model ". Com. munication présentée au Congrès Européen de l'Association des Sciences Régionales. Poitiers.

Derickl: (P.H.), 1982, Economie et planification urbaine. Vol. 2. Théories et modèles. Paris. P.U.F.

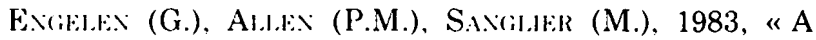
new spatial and dynamic model of an urban system ". Communication présentée au Congrès Européen de l'Association des Sciences Régionales, Poitiers.

Forrtistir (J.) 1969, Urban dynamics. Cambridge (Mass.), The M.I.T. Press (traduction française par P. Sylvestre-Baron, Paris, Economica, 1979, 329 p.).

FockNit: (S.) 1983, "Dynamic of the urban growth of a french average size-city: the CARPE model". Communication au colloque New Directions in $\mathrm{Ur}$. ban Modelling, Université de Waterloo (Ontario).

LAC'De: (C.), Folrivis (S.), 1978, Application du modèle de Forrester à la ville de Carpentras. Université de Paris X - Nanterre, Thèse de $3^{\prime \prime}$ cycle d'Economie urbaine.

L(NMB৯RI) (S.T.), RABINO (G.A.), 1983, " Non-linear dynamic models for spatial interaction : the results of some empirical experiment ". Communication présentée au Congrès Européen de l'Association des Sciences Régionales, Poitiers.

LowRy (I.) 1964, A model of metropolis. RM.4035.RC, Rand Corporation. Santa Monica.

Pricocials (I.), Stricitrsi (I.), 1979, La nouvelle alliance. Paris, Gallimard.

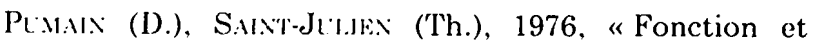
hiérarchie des villes françaises". Annales de Géographie, no 470 , p. 385-440.

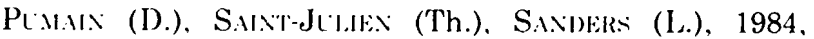
"Dynamics of spatial structure in French urban agglomerations". Papers of the Regional Science Association. à paraître.

WII.s(). (A.G.) 1970, Entropy in urban and regional modelling. London, Pion.

WILAO (A.G.) 1981. Catastrophe theory and bifurcations. Applications to urban and regional sistems. London. Croom Helm. 